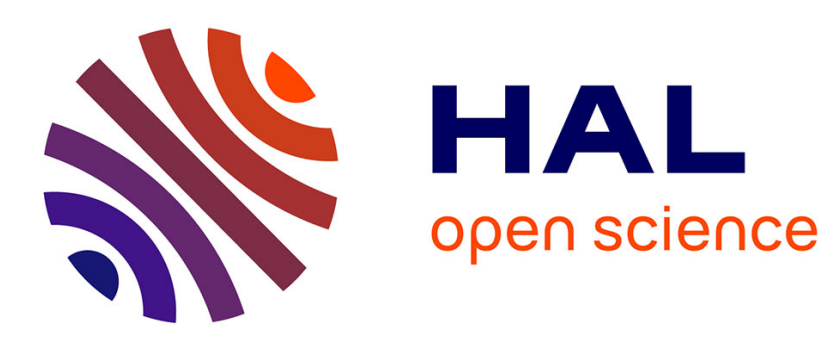

\title{
The nutraceutical potential of omega-3 alpha-linolenic acid in reducing the consequences of stroke
}

\author{
Nicolas Blondeau
}

\section{To cite this version:}

Nicolas Blondeau. The nutraceutical potential of omega-3 alpha-linolenic acid in reducing the consequences of stroke. Biochimie, 2016, 120, pp.49-55. 10.1016/j.biochi.2015.06.005 . hal-02266089

\section{HAL Id: hal-02266089 \\ https://hal.science/hal-02266089}

Submitted on 13 Aug 2019

HAL is a multi-disciplinary open access archive for the deposit and dissemination of scientific research documents, whether they are published or not. The documents may come from teaching and research institutions in France or abroad, or from public or private research centers.
L'archive ouverte pluridisciplinaire HAL, est destinée au dépôt et à la diffusion de documents scientifiques de niveau recherche, publiés ou non, émanant des établissements d'enseignement et de recherche français ou étrangers, des laboratoires publics ou privés. 
The Nutraceutical Potential of Omega-3 Alpha-Linolenic Acid in Reducing the Consequences of Stroke

\author{
${ }^{1,2}$ Nicolas Blondeau \\ ${ }^{1}$ Université de Nice Sophia Antipolis, IPMC, Sophia Antipolis, F-06560, France; \\ ${ }^{2}$ CNRS, IPMC, Sophia Antipolis, F-06560, France.
}

\author{
Corresponding Author: Nicolas Blondeau \\ Institut de Pharmacologie Moléculaires et Cellulaires \\ UMR7275, C.N.R.S, \\ 06560, Valbonne, France \\ Telephone: +33493957740 \\ Telefax: +33493957708 \\ email: Blondeau@ipmc.cnrs.fr
}

Running title: ALA importance in combating stroke

\title{
Highlights
}

ALA was evaluated as a nutraceutical in rodent model of ischemic stroke.

ALA supplementation by modification of the daily diet prevents mortality and cerebral damage.

ALA stimulates neuronal protection, neuroplasticity, and brain artery vasodilation.

ALA stimulates brain preconditioning mechanisms.

We propose the novel concept of brain preconditioning by nutraceuticals against stroke. 
Abbreviations

ALA: Alpha-Linolenic Acid

ANSES: Agence Nationale pour la Sécurité et la Santé

BDNF: Brain Derived Neurotrophic Factor

CVD: CardioVascular diseases

DHA: DocosaHexaenoic Acid

EPA: EicosaPentaenoic Acid

LA: linolenic acid

LC omega-3: long-chain omega-3 (eg, mainly EPA and DHA)

MCAO: middle cerebral artery occlusion

PUFA: polyunsaturated fatty acids

SNAP-25: Synaptosomal-Associated Protein 25

VAMP-2: Vesicle-Associated Membrane Protein 2

VGLUT1: Vesicular Glutamate Transporter 1

VGLUT2: Vesicular Glutamate Transporter 2 


\section{Abstract:}

Stroke is a worldwide major cause of mortality and morbidity. Preclinical studies have identified over 1000 molecules with brain-protective properties. More than 200 clinical trials have evaluated neuroprotective candidates for ischemic stroke yet, to date almost all failed, leading to a re-analysis of treatment strategies against stroke. An emerging view is to seek combinatory therapy, or discovering molecules able to stimulate multiple protective and regenerative mechanisms. A pertinent experimental approach to identify such candidates is the study of brain preconditioning, which refers to how the brain protects itself against ischemia and others stress-inducing stimuli. The recent discovery that nutrients like alphalinolenic acid (ALA is an essential omega-3 polyunsaturated fatty acid required as part of our daily diet), may be an efficient brain preconditionner against stroke fosters the novel concept of brain preconditioning by nutraceuticals.

This review stresses the underestimated role of nutrition in preventing and combating stroke. Although there is a consensus that increased consumption of salt, fatty foods and alcoholic beverages may promote pathologies like hypertension, obesity and alcoholism - all of which are well known risk factors of stroke - few risk factors are attributed to a deficiency in an essential nutrient in the diet. The ALA deficiency observed in the Western modern diets may itself constitute a risk factor.

This review outlines how ALA supplementation by modification of the daily diet prevented mortality and cerebral damage in a rodent model of ischemic stroke. It also describes the pleiotropic ability of ALA to trigger responses that are multicellular, mechanistically diverse, resulting in neuronal protection, stimulation of neuroplasticity, and brain artery vasodilation. Overall, this review proposes a promising therapeutic opportunity by integrating a nutritional-based approach focusing on enriching the daily diet in ALA to prevent the devastating damage caused by stroke.

\section{Keywords:}

Ischemic stroke - brain preconditioning - neuroprotection - omega-3 polyunsaturated fatty acids - functional food - synaptogenesis - neurogenesis - nutraceutical - enriched diet 


\section{Stroke is a worldwide main cause of mortality and morbidity, lacking therapeutic options}

Stroke is a devastating disease in developed and $3^{\text {rd }}$ world countries, due to its high incidence, its brutal impact on the patient and its relatives, and the lack of therapeutic options. On average, someone has a stroke every 40 and 90 seconds in the United States and Europe, respectively [1,2]. Annually, 15 million people worldwide suffer a stroke. The total number of stroke deaths is estimated at a half million people per year in European Union and is three time higher in the U.S. Of these, 30\% die and another 30\% are left permanently disabled, placing a tremendous burden on family and community. The estimated cost of stroke for 2010 was $\$ 74$ billion and $€ 64$ billion in the U.S and Europe, respectively [2, 3].

For the public, stroke is better known as brain attack, because it strikes in $85 \%$ of the cases by disrupting the blood flow to part of the brain due to occlusion of a blood vessel feeding the brain. Stroke is therefore a hypoxic-ischemic injury, whose pathophysiology involves glutamate, the major physiological excitatory neurotransmitter in the brain. The lack of oxygen and glucose causes a massive release of glutamate from neurons, and the overactivation (excitotoxicity) of ionotropic glutamate receptors, predominantly the $\mathrm{N}$ methyl-D-aspartate (NMDA) glutamate receptor subtype. This results in accumulation of intracellular calcium, which in turn triggers deleterious cascades including activation of lytic enzymes, mitochondrial dysfunction, oxidative stress and inflammation [4] in two regions that coexist within the infarct: the necrotic core and the ischemic penumbra, an area surrounding the core where neurons remain on the brink of survival or death for hours [5].

The progresses in understanding its complex interplay of multiple cellular and signaling pathways that alter the neurovascular unit integrity within differentially affected territories allowed identification in preclinical studies of neuroprotective targets or/and drugs blocking the neurotoxic ischemic cascade. Nevertheless, of those tested in clinical trials, all have failed, leaving patients and clinicians without any repertoire of therapeutic opportunities exerting direct protection of the neurons [6]. Consequently, the only approved therapeutic exerts its benefits through the restoration of the blood flow to the brain by blood clot disruption. It is performed by recombinant tissue plasminogen activator (tPA) treatment administered to approximately $5 \%$ of stroke patients. On the positive side, three definitive points are worth noting: 1) Efforts have been made over the past decades in high-income countries to control 
major risk factors like hypertension, diabetes, and high cholesterol, strides which have contributed to stroke mortality reduction. This success is associated with a global improvement in population health. It should be monitored cautiously as it probably represents the tip of the iceberg because stroke mortality represents, at the maximum, a third of the annual first-ever strokes; 2) The failure in translation from experimental models to clinical trials has lead to revisiting the research of strategies against stroke, resulting in a set of drug development criteria, collectively known as the Stroke Therapy Academic Industry Roundtable (STAIR) recommendations [7]; and 3) There is an acceleration in the study of unconventional therapies, with a major theme being to interrogate how the brain protects itself $[4,8]$.

To summarize, considering the multifactorial nature of stroke, in order to be considered as a good candidate for a clinical trial, a treatment should exhibit multimodal actions on the multiple cell types composing the neurovascular unit. Consequently, an emerging view is that we should preferentially seek and test for drug combinations or multi-therapy, or discovering molecules able to stimulate multiple protective and regenerative mechanisms to fight stroke $[9,10]$. A pertinent experimental approach to identify such candidates came from our experience on brain preconditioning through the study of how the brain protects itself against ischemia and others stress stimuli.

\section{The study of the brain preconditioning opens new rational against stroke}

The idea of developing a treatment against stroke inspired by this endogenous protective process is appealing. Preconditioning depends on the stimulation of protection and regeneration against stroke through direct and/or indirect mechanisms, involving multiple cell types, rather than through inhibition of single deleterious events, or targets of most of the conventional neuroprotective approaches. Indeed, brain preconditioning refers to a sublethal toxic stimulus eliciting an endogenous response, which renders the brain remarkably tolerant to a subsequent, normally lethal stimulus of the same insult. Since its original description in the brain [11], the discovery that non-ischemic preconditioners (Figure 1) including various sublethal insults like epilepsy, endotoxins, anoxia, hyperthermia and spreading depression also promote tolerance to ischemia - a phenomenon known as "cross-tolerance" $[8,12,13]$ definitely established that the protective response to brain preconditioners is pleiotropic in nature. A major conceptual roadblock for clinical translation - the requirement of bringing 
neurons to the 'brink of death' during the sublethal preconditioning challenge [14] - can be circumvented based on the demonstration that brain preconditioning may be pharmacologically/chemically induced by drugs like adenosine or $\mathrm{K}_{\mathrm{ATP}}$ channel agonists [15]. Finally, the recent discovery that nutrients like polyunsaturated fatty acids and lysophospholipids that form part of our daily diet may be efficient brain preconditionners against stroke $[16,17]$ gave birth to the novel concept of brain preconditioning based on nutraceuticals against stroke [18].

\section{The novel concept of brain preconditioning by nutraceuticals against stroke stresses that nutritional importance may go beyond stroke prevention}

The rationale of supplementation with a non-ischemic preconditioner that could be a natural product - a nutrient defined as a nutraceutical - is based on the experimental demonstration that certain nutraceuticals can act as "natural preconditioners" to increase brain resistance against stroke [18]. Such an approach circumvents issues such as the administration routes and timing issues which thus far have plagued the preconditioning and neuroprotectant fields; such an approach may be more amenable for translation to the clinical arena, since nutrition plays a key role for health and stroke risk in particular. Indeed, most modifiable risk factors of stroke - including hypertension, diabetes, hypercholesterolemia, tobacco use, increased inflammatory markers, dyslipidemia and obesity - often coexist with improper life-style and nutrition, causing imbalances in essential vitamins and nutriments. Striking examples are over-consumption of salt, fatty acids that promotes hypertension and diabetes/obesity that drastically increase the possibility of having a stroke. In contrast, epidemiologic studies usually do not clearly identify a risk factor arising from a deficiency in an essential nutrient in the diet. Nevertheless, an important exception is that many clinical and epidemiologic studies have shown that insufficient dietary intake of fruits and vegetables or of foods containing omega-3 polyunsaturated fatty acids (PUFAs), in the form of Alpha-Linolenic Acid (ALA) and the Long Chain derivatives (LC-n-3), Eicosa-Pentaenoic-Acid (EPA) and DocosaHexaenoic-Acid (DHA), represent a risk factor for cardiovascular and cerebral diseases, including coronary heart disease and stroke. In addition, several studies investigating the importance of dietary omega-3 PUFAs, achieved by consumption of seafood (rich in LC-n-3: EPA and DHA) and/or vegetable oils rich in precursor (ALA), proposed omega-3 PUFAs as key support for neurons and brain resistance (for review, see [18, 19]. Therefore, nutritional products with health benefits or supplemented with such protective nutrients seem promising 
for reducing the incidence of stroke, encouraging the discovery or characterization of an efficient nutraceutical targeted against stroke.

Unfortunately, no such entity like the STAIR recommendations or any other guidelines exist to directly address nutraceutical discovery, especially in the field of stroke. Nutraceutical is a termed coined from nutrition and pharmaceutical without any regulatory definition, and refers to foods or one of its constituents that provides medical or health benefits, including the prevention and/or treatment of a disease [20]. Such an open definition may explain, in part, why the term is often abused for marketing purposes, and several limitations exist in proving the efficiency of a potential candidate to be termed nutraceutical.

The main distinction between nutraceutical and dietary supplements aimed at supporting the body with the required amount of a certain nutrient needed for its proper functioning, is that a nutraceutical should have a proven efficiency against disease [20]. Thus, a supplement may be a nutraceutical;, such overlap increases the risk of confusion, especially if the essential distinction of the role of the nutraceutical in combating disease is not well framed. To avoid repeating past mistakes in the field, the best chance for success may be to move forward with a nutraceutical being able to follow, at least for part, the STAIR recommendations. This goal would be achievable by restricting the definition of the term nutraceutical to compounds isolated or purified from foods, and demonstrating that efficiency could be evaluated and used in medicinal forms. In this framework, the following part of the review argues that AlphaLinolenic Acid conforms to this optimal definition of nutraceutical, by identifying its relevance in protecting from stroke consequence.

\section{Omega-3 supplementation to lower stroke risk: origin of the concept}

A body of evidence shows that omega- 3 intake in the adult population of developed countries is far below the recommended Dietary Reference Intake. This deficiency was associated with an increased risk of stroke occurrence and conversely, diets enriched in omega-3, especially through the consumption of fatty fish twice a week, lower the risk of having a stroke [21, 22]. The first clinical trials examining the effect of fish oil EPA-DHA supplementation on cardiovascular death were promising, indicating that omega-3 supplementation may be used for lowering stroke risk $[23,24]$. That conclusion is disputed by results from recent randomized clinical trials examining the effects of fish oil supplementation on cardiovascular 
disease morbidity and mortality in secondary prevention settings [25, 26]. Nonetheless, French recommendations, pointing out the necessity to enrich our daily diet in omega-3 fatty acids [27-30].

Additionally, the few preclinical studies, which have examined the effects of fish oil supplementation in rodent models of ischemic brain injury, suggested a protective action against neuronal damage (for review, see $[18,19,31]$ ). It is important to note that beneficial effects of LC-omega-3 enriched diets were mainly observed when comparing with diets lacking EPA/DHA. This may explain in part the inconsistency of recent results of clinical trials investigating the effect of omega-3 supplementation: the protection obtained with supplementation with LC-omega-3 may only appear in the case of a severe deficiency in the patient diet. This reinforces the necessity for a clear distinction between nutraceuticals and supplements that should exert a beneficial role compensating a deficiency of the intake. In the context of investigating omega- 3 as a nutraceutical, protection of the patients by the enriched diet should also be obtained when comparing to patients fed a diet already containing omega3 fatty acids of the same nature. Such nutraceutical effect of ALA was discretely suggested in the "Lyon Diet Heart Study", which reported a reduced rate of recurrence of myocardial infarction, other cardiac events and overall mortality of patients fed an ALA-rich diet as compared to those fed the usual post-infarct diet, known as the "prudent diet" that also contains ALA and other omega-3 fatty acids [32].

\section{Alpha-linolenic acid: from supplements to nutraceuticals for stroke?}

\subsection{Long underestimated, ALA interest as supplement is now re-evaluated}

Indeed, while numerous studies have investigated the beneficial effect of a DHA/EPAenriched diet, as well as providing characterization of biophysical and functional properties of the nutrient itself, interest in ALA is extremely recent. Several reasons for this early disinterest can be surmised. The first reason was conceptual: DHA is a major constituent of the brain plasma membranes but ALA is not even incorporated at the brain membrane level. In addition, its consumption may not fulfill DHA requirements [33], because of its extremely low efficiency of conversion to DHA [34, 35]. This dogma is now disputed because, as reviewed by Barcelo-Coblijn, when consumed in adequate amounts over time, ALA exerts 
identical effects as DHA in several physiological processes [33]. The second reason resulted diseases (CVD incidence is lower for an EPA and DHA combination compared to ALA [36, 37]). Consequently to the recent controversy on the effect of supplementation performed with EPA or DHA on stroke and CVD and to the growing evidence of the beneficial effect of dietary ALA to protect against CVD, dietary supplementation with ALA regained interest.

A recent body of evidence demonstrates that ALA intake is associated with a reduced risk of stroke in humans. It is worth noting that this effect on stroke was not correlated with a positive effect on coronary heart disease [38]. A reduced risk of stroke was associated with high serum levels of ALA [39]. A lower prevalence of a carotid plaque and inflammation, key steps of atherosclerosis considered as one of the major risk factors of stroke, are associated with elevated intake of ALA [40-42]. The interest in ALA as a supplement is also reinforced by the fact that human do not possess the enzymes for de novo synthesis of ALA, in contrast to EPA/DHA; and by the absolute and relative recent changes of omega-6/omega-3 ratio that seems mainly mediated by an increase in LA and a decrease in ALA content in the western $\operatorname{diet}[43]$

\subsection{ALA as a nutraceutical for stroke: proof of concept}

Just as for the beneficial effects of an LC-omega-3 enriched diet (observed when comparing with diets deficient in EPA/DHA), a diet rich in ALA from perilla oil increases lifetimes of hypertensive stroke-prone rats compared to a diet rich in LA from sunflower oil [44]. To avoid any confusion between the concept of a supplement and a nutraceutical, we investigated whether an ALA enriched diet from rapeseed oil, a rich source of ALA and the only source of lipids, could reduce brain damage in a mouse model of ischemic stroke (Figure 2), performed by transient occlusion of the middle cerebral artery (MCAO), compared to regular chows supplemented in ALA, EPA and DHA in proportions matching the "murine" recommended intake $[45,46]$. In addition, the ALA enriched diet did not contain any LC-omega-3 EPA or DHA. In this paradigm of a 6-week diet enriched in ALA by a factor of three compared to regular chows, a reduced mortality rate and smaller infarct size were observed $24 \mathrm{~h}$ after $60 \mathrm{~min}$ of MCAO [47]. The reduction of the infarct volume was similar or better than those observed with high dietary levels of LNC omega-3 provided over the same period of feeding 
[48]. Since ALA bioconversion to DHA was negligible over the period of investigation, this neuroprotection may be attributed to a potential nutraceutical effect of ALA.

\subsection{ALA evaluated and used in medicinal forms in rodent model of ischemic stroke}

As reviewed above, the main distinction between nutraceuticals and dietary supplements is that the proven efficiency on disease was observed - at least - in a mice model of stroke. Thus, the additional step was to investigate the hypothesis that the use of ALA could conform to this restricted definition of a nutraceutical, where efficiency could be evaluated when used in medicinal treatment. Evidence has accumulated that bolus injections of ALA and, to a lesser extent, EPA/DHA, is neuroprotective against glutamate-mediated excitotoxicity, a major cause of the initial substantial neuronal damage in animal models of neurodegenerative conditions and neurological injury, like epileptic seizures $[49,50]$, acute spinal cord injury $[31,51,52]$ and focal and global ischemia $[17,50,52-57]$.

With regard to acute neuroprotection, pretreatment and "rapid preconditioning" when targeting glutamate excitotoxicity share the same temporal constraint that undermines clinical translation. Nevertheless, testing ALA in such a paradigm provided the first indication of its nutraceutical capacity as a neuroprotectant. The injection of ALA (i.c.v., 10 $\mu \mathrm{M} / 5 \mu 1$ or i.v., $500 \mathrm{nmol} / \mathrm{kg}$ ) preserved $80 \%$ of the CA1 hippocampal pyramidal neurons in an in vivo transient model of global ischemia, a model for which neuronal death is mainly driven by glutamate excitotoxicity, as compared to the $15 \%$ survival observed 7 days after 20 min of the 4-vessel occlusion [50]. In the MCAO model (Figure 2), intravenous injection of ALA reduced the infarct volume when injected before, but also up to 6 hours after stroke onset, corresponding with post-treatment or postconditioning paradigms [56, 58]. In such a context of standardized evaluation of a neuroprotectant candidate, ALA performed like riluzole, a drug currently in clinical use for amyotrophic lateral sclerosis in a time-frame of intervention compatible within a clinical setting.

When considering nutraceuticals, an interesting aspect offered by the preconditioning and postconditioning approaches is that repetitive activation of these endogenous mechanisms may lead to sustained protection against ischemic stroke [59, 60]. Such an approach with repeated intravenous injections achieved a 3-fold improvement of the long-term survival postischemia, while no improvement was observed with a single ALA injection - described to 
reduce post-stroke infarct $[56,58]$. The surprising number of neuronal death models and

\subsection{The multiple temporal and mechanistic protective effects of ALA}

The original mechanistic finding was that ALA can act directly on the neurons by protecting them in vitro of neuronal death triggered by excitotoxicity driven by exposure to a $\mathrm{Mg}^{2+}$ depleted glycine-supplemented medium $(-\mathrm{Mg} /+\mathrm{gly})$ or the addition of an excitotoxic concentration of glutamate [50,58]. The second was that ALA also acts on another cell type comprising the neurovascular unit, namely the endothelial cells. Indeed, neuroprotective doses of ALA display vasoactive properties. Ex vivo, ALA increased the diameter of the basilar but not carotid artery in mice and rats [53], leading to an approximately $30 \%$ increase in artery diameter, which could account for an increase of the CBF observed in vivo within 30 min after injection of a neuroprotective dose of ALA [53]. The third mechanistic finding was the ability of ALA to stimulate neuronal plasticity. The reduced long-term mortality rate observed with repeated ALA injections spaced over several days implied additional protective mechanisms other than solely reducing glutamate excitotoxicity within the acute phase of stroke. We have directly investigated whether ALA may stimulate spontaneous biological functions that are implicated in stroke recovery and targeted by restorative therapies. Subchronic ALA treatment induces neurogenesis in the dentate gyrus, identified by an increased number of proliferating immature neurons 3 days after the final ALA injection[58]. Those immature neurons became mature by 21 days. The concept that ALA improved neuronal plasticity was strengthened by the discovery that synaptogenesis was concomitantly stimulated by the ALA treatment, as shown by up-regulation of key proteins involved in synaptic function (synaptophysin-1, VAMP-2, and SNAP-25) as well as proteins supporting glutamatergic neurotransmission (V-GLUT1 and V-GLUT2). In many studies, stimulation of neurogenesis and synaptogenesis implied an up-regulation of neurotrophic factors such as Brain Derived Neurotrophic Factor (BDNF), for which beneficial effects on stroke have been widely described [61]. These changes also correlated with an increase in BDNF protein levels, both in vivo, following sub-chronic ALA treatment. Similar results were observed in vitro when applying ALA on neural stem cells and hippocampal cultures [58]. With regard to stroke management, the protective actions identified with ALA conform with its role as a 
treatment for stroke, taking in consideration the necessity of a multimodal action mode on the major mechanistic finding that explains the new concept of nutraceutical being a natural brain preconditioner is the demonstration that ALA is a "natural" preconditioner able to induce delayed cerebral tolerance to ischemia.

\subsection{ALA a nutraceutical stimulating brain preconditioning mechanisms}

The capacity of ALA as a non-ischemic preconditioner able to induce brain tolerance against excitotoxicity-driven neuronal death was demonstrated in rat models of global ischemia and kainic acid-induced epileptic seizures [17]. ALA preconditioning was also successfully demonstrated in a mouse model of transient focal ischemia [58]. The temporal window of brain protection and the protective pathways triggered by ALA preconditioning paralleled preconditioning by sublethal insults and by adenosine and $\mathrm{K}_{\mathrm{ATP}}$ channel agonists that are acknowledged as gold standards in chemical preconditionings [15, 17, 54]. ALA preconditioning induced the neuroprotective HSP70 heat shock protein and the expression/activation of the transcription factor nuclear factor-kB (NFkB) within a similar time frame and neuronal localization shared by ischemic, epileptic and chemical preconditionings $[15,17,54]$. Such similarities clearly establish ALA as a nutraceutical in its capacity to trigger the ubiquitous pleiotropic protective mechanisms of brain preconditioning.

\section{Conclusion}

This review presents the capacity of alpha-linolenic acid, the precursor of the LComega-3 polyunsaturated fatty acid, for protecting the brain from stroke. This review highlights its interest as a supplement to reduce the incidence of stroke and as a nutraceutical to increase brain resistance against stroke damage, in both nutritional and medicinal forms. The concept underlying the nutraceutical potential of ALA is its pleiotropic targeting of many brain cell types, leading not only to inhibition of the deleterious pathways driven by glutamate excitotoxicity but also to enhance restorative mechanisms such as neurotrophic factor production, neurogenesis and synaptogenesis. The examples of multiple actions of ALA illustrate the current importance of reaching a consensus definition of nutraceutical required for shaping future research in the field. This point seems mandatory because the characterization and use of a nutraceutical like ALA represents a paradigm shift, from 
focusing solely on the discovery of new drugs designed for clinical neuroprotection, to the identification of therapeutic capacity of natural food constituents. This is of importance because 1) the medicinal forms of a nutraceuticals may be of advantage as compared to drugs or non-natural preconditioners that display ceiling effects (dose/duration) due to their toxic side effects; 2) development of nutraceuticals against stroke may largely depend not only upon its successful translation to the clinical arena, but also to daily life, notably through functional food development. Such a goal opens an interesting avenue - supported by the characteristics of ALA as a preconditioner - that may be achieved by integrating knowledge drawn from preconditioning research. Though extremely relevant for stroke, this novel concept of nutraceutical preconditioning may not be restricted to ALA or stroke, but may in fact be extend to other existing or novel nutraceuticals and neuropathologies and neurovascular diseases. Hence, the overall effect of ALA, including its capacity to trigger preconditioning, appears to be useful for shaping new recommendations in the nutritional as well as therapeutic approach of stroke, thereby justifying continued investigation in other traumas, epilepsy, SCI and neurodegenerative insults that have been shown to be protected by pre- and post-conditioning. 


\section{Acknowledgments}

Ideas discussed here are based in part on the author's presentation at the 12th Euro Fed Lipid congress held in Montpellier in September 2014. I am grateful to CNRS, the Fondation pour la Recherche Médicale (grant number DRM20101220421), ONIDOL and St Hubert for their support. I wish to thank Dr Joseph Tauskela for his critical reading of the manuscript and Franck Aguila for his technical assistance with the figures. I am also grateful to Dr Catherine Heurteaux for many helpful discussions and to all our past and present team members who have contributed to the data discussed in the review.

\section{Conflicts of interest}

None

\section{Legend of the figures}

Figure 1: The different natures of brain preconditioners and their perspectives against stroke

Originally, brain preconditioning refers to an endogenous response to a sublethal stimulus in the brain, which develops tolerance to a subsequent, normally lethal stimulus of the same insult. The protective response to brain preconditioners is pleiotropic in nature. Non-ischemic preconditioners include various sublethal insults, pharmacological/chemical agents and natural compounds including nutrients that are part of our daily diet. The diet aspect provides the rationale of supplementation with a non-ischemic preconditioner that could be a natural product - a nutrient defined as a nutraceutical.

\section{Figure 2: Alpha-Linolenic Acid (ALA) as nutraceutical for stroke: proof of concept}

The main distinction between nutraceutical and dietary supplements aimed at supporting the body with the required amount of a certain nutrient required for proper function is that a nutraceutical should have demonstrated a proven efficiency against disease. Alpha-linolenic acid fulfills the restricted definition of the term nutraceutical to compounds isolated or purified from foods, and that efficiency could be evaluated, thereby potentially being prescribed in medicinal forms. 
A nutritional approach with an ALA enriched diet from rapeseed oil (left panel) and a medicinal approach with intravenous injection of ALA (right panel) reduce brain damage in a mouse model of ischemic stroke. 


\section{References}

[1] A.S. Go, D. Mozaffarian, V.L. Roger, E.J. Benjamin, J.D. Berry, M.J. Blaha, S. Dai, E.S. Ford, C.S. Fox, S. Franco, H.J. Fullerton, C. Gillespie, S.M. Hailpern, J.A. Heit, V.J. Howard, M.D. Huffman, S.E. Judd, B.M. Kissela, S.J. Kittner, D.T. Lackland, J.H. Lichtman, L.D. Lisabeth, R.H. Mackey, D.J. Magid, G.M. Marcus, A. Marelli, D.B. Matchar, D.K. McGuire, E.R. Mohler, 3rd, C.S. Moy, M.E. Mussolino, R.W. Neumar, G. Nichol, D.K. Pandey, N.P. Paynter, M.J. Reeves, P.D. Sorlie, J. Stein, A. Towfighi, T.N. Turan, S.S. Virani, N.D. Wong, D. Woo, M.B. Turner, Heart disease and stroke statistics--2014 update: a report from the American Heart Association, Circulation 129 (2014) e28-e292.

[2] A. Gustavsson, M. Svensson, F. Jacobi, C. Allgulander, J. Alonso, E. Beghi, R. Dodel, M. Ekman, C. Faravelli, L. Fratiglioni, B. Gannon, D.H. Jones, P. Jennum, A. Jordanova, L. Jonsson, K. Karampampa, M. Knapp, G. Kobelt, T. Kurth, R. Lieb, M. Linde, C. Ljungcrantz, A. Maercker, B. Melin, M. Moscarelli, A. Musayev, F. Norwood, M. Preisig, M. Pugliatti, J. Rehm, L. Salvador-Carulla, B. Schlehofer, R. Simon, H.C. Steinhausen, L.J. Stovner, J.M. Vallat, P. Van den Bergh, J. van Os, P. Vos, W. Xu, H.U. Wittchen, B. Jonsson, J. Olesen, Cost of disorders of the brain in Europe 2010, Eur Neuropsychopharmacol 21 (2011) 718779.

[3] D. Lloyd-Jones, R.J. Adams, T.M. Brown, M. Carnethon, S. Dai, G. De Simone, T.B. Ferguson, E. Ford, K. Furie, C. Gillespie, A. Go, K. Greenlund, N. Haase, S. Hailpern, P.M. Ho, V. Howard, B. Kissela, S. Kittner, D. Lackland, L. Lisabeth, A. Marelli, M.M. McDermott, J. Meigs, D. Mozaffarian, M. Mussolino, G. Nichol, V.L. Roger, W. Rosamond, R. Sacco, P. Sorlie, T. Thom, S. Wasserthiel-Smoller, N.D. Wong, J. Wylie-Rosett, Heart disease and stroke statistics--2010 update: a report from the American Heart Association, Circulation 121 (2010) e46-e215.

[4] C. Iadecola, J. Anrather, Stroke research at a crossroad: asking the brain for directions, Nature neuroscience 14 (2011) 1363-1368.

[5] M. Fisher, B. Bastan, Identifying and utilizing the ischemic penumbra, Neurology 79 (2012) S79-85.

[6] V.E. O'Collins, M.R. Macleod, G.A. Donnan, L.L. Horky, B.H. van der Worp, D.W. Howells, 1,026 experimental treatments in acute stroke, Ann Neurol 59 (2006) 467-477.

[7] M. Fisher, Recommendations for advancing development of acute stroke therapies: Stroke Therapy Academic Industry Roundtable 3, Stroke 34 (2003) 1539-1546. 
[8] J.S. Tauskela, N. Blondeau, Prescription for stroke: Should preconditioning be investigated as a drug?, in: B.J. Schaller (Ed.), Ischemic Tolerance of the Brain, Research Signpost, Research Signpost, Kerala, India, 2009, pp. 85-135.

[9] E.H. Lo, Experimental models, neurovascular mechanisms and translational issues in stroke research, British journal of pharmacology 153 Suppl 1 (2008) S396-405.

[10] J. Minnerup, W.R. Schabitz, Multifunctional actions of approved and candidate stroke drugs, Neurotherapeutics 6 (2009) 43-52.

[11] K. Kitagawa, M. Matsumoto, M. Tagaya, R. Hata, H. Ueda, M. Niinobe, N. Handa, R. Fukunaga, K. Kimura, K. Mikoshiba, et al., 'Ischemic tolerance' phenomenon found in the brain, Brain Res 528 (1990) 21-24.

[12] J.M. Gidday, Cerebral preconditioning and ischaemic tolerance, Nat Rev Neurosci 7 (2006) 437-448.

[13] H. Plamondon, N. Blondeau, C. Heurteaux, M. Lazdunski, Mutually protective actions of kainic acid epileptic preconditioning and sublethal global ischemia on hippocampal neuronal death: involvement of adenosine A1 receptors and K(ATP) channels, J Cereb Blood Flow Metab 19 (1999) 1296-1308.

[14] U. Dirnagl, R.P. Simon, J.M. Hallenbeck, Ischemic tolerance and endogenous neuroprotection, Trends Neurosci 26 (2003) 248-254.

[15] N. Blondeau, H. Plamondon, C. Richelme, C. Heurteaux, M. Lazdunski, K(ATP) channel openers, adenosine agonists and epileptic preconditioning are stress signals inducing hippocampal neuroprotection, Neuroscience 100 (2000) 465-474.

[16] N. Blondeau, I. Lauritzen, C. Widmann, M. Lazdunski, C. Heurteaux, A potent protective role of lysophospholipids against global cerebral ischemia and glutamate excitotoxicity in neuronal cultures, J Cereb Blood Flow Metab 22 (2002) 821-834.

[17] N. Blondeau, C. Widmann, M. Lazdunski, C. Heurteaux, Polyunsaturated fatty acids induce ischemic and epileptic tolerance, Neuroscience 109 (2002) 231-241.

[18] N. Blondeau, J.S. Tauskela, A New Future in Brain Preconditioning Based on Nutraceuticals: A Focus on a-Linolenic Omega-3 Fatty Acid for Stroke Protection, in: J.M. Gidday, M.A. Perez-Pinzon, J.H. Zhang (Eds.), Innate Tolerance in the CNS, Springer New York New York, 2013, pp. 133-163.

[19] C. Nguemeni, E. Gouix, M. Bourourou, C. Heurteaux, N. Blondeau, Alpha-linolenic acid: A promising nutraceutical for the prevention of stroke, PharmaNutrition 1 (2013) 1-8.

[20] E.K. Kalra, Nutraceutical--definition and introduction, AAPS PharmSci 5 (2003) E25. 
[21] K. He, Y. Song, M.L. Daviglus, K. Liu, L. Van Horn, A.R. Dyer, P. Greenland,

Accumulated evidence on fish consumption and coronary heart disease mortality: a metaanalysis of cohort studies, Circulation 109 (2004) 2705-2711.

[22] J.C. Stanley, R.L. Elsom, P.C. Calder, B.A. Griffin, W.S. Harris, S.A. Jebb, J.A. Lovegrove, C.S. Moore, R.A. Riemersma, T.A. Sanders, UK Food Standards Agency Workshop Report: the effects of the dietary n-6:n-3 fatty acid ratio on cardiovascular health, Br J Nutr 98 (2007) 1305-1310.

[23] G.I.p.l.S.d.S.n.I. miocardico, Dietary supplementation with n-3 polyunsaturated fatty acids and vitamin E after myocardial infarction: results of the GISSI-Prevenzione trial., Lancet 354 (1999) 447-455.

[24] M. Yokoyama, H. Origasa, M. Matsuzaki, Y. Matsuzawa, Y. Saito, Y. Ishikawa, S. Oikawa, J. Sasaki, H. Hishida, H. Itakura, T. Kita, A. Kitabatake, N. Nakaya, T. Sakata, K. Shimada, K. Shirato, Effects of eicosapentaenoic acid on major coronary events in hypercholesterolaemic patients (JELIS): a randomised open-label, blinded endpoint analysis, Lancet 369 (2007) 1090-1098.

[25] A. Messori, V. Fadda, D. Maratea, S. Trippoli, omega-3 fatty acid supplements for secondary prevention of cardiovascular disease: from "no proof of effectiveness" to "proof of no effectiveness", JAMA Intern Med 173 (2013) 1466-1468.

[26] S.M. Kwak, S.K. Myung, Y.J. Lee, H.G. Seo, Efficacy of omega-3 fatty acid supplements (eicosapentaenoic acid and docosahexaenoic acid) in the secondary prevention of cardiovascular disease: a meta-analysis of randomized, double-blind, placebo-controlled trials, Arch Intern Med 172 (2012) 686-694.

[27] R.M. Krauss, R.H. Eckel, B. Howard, L.J. Appel, S.R. Daniels, R.J. Deckelbaum, J.W. Erdman, Jr., P. Kris-Etherton, I.J. Goldberg, T.A. Kotchen, A.H. Lichtenstein, W.E. Mitch, R. Mullis, K. Robinson, J. Wylie-Rosett, S. St Jeor, J. Suttie, D.L. Tribble, T.L. Bazzarre, AHA Dietary Guidelines: revision 2000: A statement for healthcare professionals from the Nutrition Committee of the American Heart Association, Circulation 102 (2000) 2284-2299.

[28] N.J. Stone, Fish consumption, fish oil, lipids, and coronary heart disease, Circulation 94 (1996) 2337-2340.

[29] P.M. Kris-Etherton, W.S. Harris, L.J. Appel, Fish consumption, fish oil, omega-3 fatty acids, and cardiovascular disease, Circulation 106 (2002) 2747-2757.

[30] A. Martin, The "apports nutritionnels conseilles (ANC)" for the French population, Reprod Nutr Dev 41 (2001) 119-128. 
[31] A.T. Michael-Titus, Omega-3 fatty acids and neurological injury, Prostaglandins Leukot Essent Fatty Acids 77 (2007) 295-300.

[32] M. de Lorgeril, P. Salen, Alpha-linolenic acid and coronary heart disease, Nutr Metab Cardiovasc Dis 14 (2004) 162-169.

[33] G. Barcelo-Coblijn, E.J. Murphy, Alpha-linolenic acid and its conversion to longer chain n-3 fatty acids: benefits for human health and a role in maintaining tissue n-3 fatty acid levels, Prog Lipid Res 48 (2009) 355-374.

[34] N. Hussein, E. Ah-Sing, P. Wilkinson, C. Leach, B.A. Griffin, D.J. Millward, Longchain conversion of [13C]linoleic acid and alpha-linolenic acid in response to marked changes in their dietary intake in men, J Lipid Res 46 (2005) 269-280.

[35] R.J. Pawlosky, J.R. Hibbeln, J.A. Novotny, N. Salem, Jr., Physiological compartmental analysis of alpha-linolenic acid metabolism in adult humans, J Lipid Res 42 (2001) 1257-1265.

[36] G.C. Burdge, P.C. Calder, Dietary alpha-linolenic acid and health-related outcomes: a metabolic perspective, Nutrition research reviews 19 (2006) 26-52.

[37] C. Wang, W.S. Harris, M. Chung, A.H. Lichtenstein, E.M. Balk, B. Kupelnick, H.S. Jordan, J. Lau, n-3 Fatty acids from fish or fish-oil supplements, but not alpha-linolenic acid, benefit cardiovascular disease outcomes in primary- and secondary-prevention studies: a systematic review, Am J Clin Nutr 84 (2006) 5-17.

[38] J. de Goede, W.M. Verschuren, J.M. Boer, D. Kromhout, J.M. Geleijnse, Alphalinolenic Acid intake and 10-year incidence of coronary heart disease and stroke in 20,000 middle-aged men and women in the Netherlands, PLoS One 6 (2011) e17967.

[39] J.A. Simon, J. Fong, J.T. Bernert, Jr., W.S. Browner, Serum fatty acids and the risk of stroke, stroke 26 (1995) 778-782.

[40] G. Zhao, T.D. Etherton, K.R. Martin, P.J. Gillies, S.G. West, P.M. Kris-Etherton, Dietary alpha-linolenic acid inhibits proinflammatory cytokine production by peripheral blood mononuclear cells in hypercholesterolemic subjects, Am J Clin Nutr 85 (2007) 385391.

[41] F.M. van der Valk, D.F. van Wijk, E.S. Stroes, Novel anti-inflammatory strategies in atherosclerosis, Curr Opin Lipidol 23 (2012) 532-539.

[42] L. Djousse, S.C. Hunt, D.K. Arnett, M.A. Province, J.H. Eckfeldt, R.C. Ellison, Dietary linolenic acid is inversely associated with plasma triacylglycerol: the National Heart, Lung, and Blood Institute Family Heart Study, Am J Clin Nutr 78 (2003) 1098-1102. 
[43] A.P. Simopoulos, Overview of evolutionary aspects of omega 3 fatty acids in the diet, World Rev Nutr Diet 83 (1998) 1-11.

[44] T. Shimokawa, A. Moriuchi, T. Hori, M. Saito, Y. Naito, H. Kabasawa, Y. Nagae, M. Matsubara, H. Okuyama, Effect of dietary alpha-linolenate/linoleate balance on mean survival time, incidence of stroke and blood pressure of spontaneously hypertensive rats, Life sciences 43 (1988) 2067-2075.

[45] J.M. Bourre, O. Dumont, G. Pascal, G. Durand, Dietary alpha-linolenic acid at 1.3 $\mathrm{g} / \mathrm{kg}$ maintains maximal docosahexaenoic acid concentration in brain, heart and liver of adult rats, J Nutr 123 (1993) 1313-1319.

[46] C. Pudelkewicz, J. Seufert, R.T. Holman, Requirements of the female rat for linoleic and linolenic acids, J Nutr 94 (1968) 138-146.

[47] C. Nguemeni, B. Delplanque, C. Rovere, N. Simon-Rousseau, C. Gandin, G. Agnani, J.L. Nahon, C. Heurteaux, N. Blondeau, Dietary supplementation of alpha-linolenic acid in an enriched rapeseed oil diet protects from stroke, Pharmacol Res 61 (2010) 226-233.

[48] J.K. Relton, P.J. Strijbos, A.L. Cooper, N.J. Rothwell, Dietary N-3 fatty acids inhibit ischaemic and excitotoxic brain damage in the rat, Brain Res Bull 32 (1993) 223-226.

[49] R.A. Voskuyl, M. Vreugdenhil, J.X. Kang, A. Leaf, Anticonvulsant effect of polyunsaturated fatty acids in rats, using the cortical stimulation model, Eur J Pharmacol 341 (1998) 145-152.

[50] I. Lauritzen, N. Blondeau, C. Heurteaux, C. Widmann, G. Romey, M. Lazdunski, Polyunsaturated fatty acids are potent neuroprotectors, EMBO J 19 (2000) 1784-1793.

[51] V.R. King, W.L. Huang, S.C. Dyall, O.E. Curran, J.V. Priestley, A.T. Michael-Titus, Omega-3 fatty acids improve recovery, whereas omega- 6 fatty acids worsen outcome, after spinal cord injury in the adult rat, J Neurosci 26 (2006) 4672-4680.

[52] L. Lang-Lazdunski, N. Blondeau, G. Jarretou, M. Lazdunski, C. Heurteaux, Linolenic acid prevents neuronal cell death and paraplegia after transient spinal cord ischemia in rats, $\mathbf{J}$ Vasc Surg 38 (2003) 564-575.

[53] N. Blondeau, O. Petrault, S. Manta, V. Giordanengo, P. Gounon, R. Bordet, M. Lazdunski, C. Heurteaux, Polyunsaturated fatty acids are cerebral vasodilators via the TREK1 potassium channel, Circulation research 101 (2007) 176-184.

[54] N. Blondeau, C. Widmann, M. Lazdunski, C. Heurteaux, Activation of the nuclear factor-kappaB is a key event in brain tolerance, J Neurosci 21 (2001) 4668-4677. 
[55] C. Heurteaux, N. Guy, C. Laigle, N. Blondeau, F. Duprat, M. Mazzuca, L. LangLazdunski, C. Widmann, M. Zanzouri, G. Romey, M. Lazdunski, TREK-1, a K+ channel involved in neuroprotection and general anesthesia, EMBO J 23 (2004) 2684-2695.

[56] C. Heurteaux, C. Laigle, N. Blondeau, G. Jarretou, M. Lazdunski, Alpha-linolenic acid and riluzole treatment confer cerebral protection and improve survival after focal brain ischemia, Neuroscience 137 (2006) 241-251.

[57] Y.F. Xiao, S.N. Wright, G.K. Wang, J.P. Morgan, A. Leaf, Fatty acids suppress voltage-gated $\mathrm{Na}+$ currents in HEK293t cells transfected with the alpha-subunit of the human cardiac Na+ channel, Proc Natl Acad Sci U S A 95 (1998) 2680-2685.

[58] N. Blondeau, C. Nguemeni, D.N. Debruyne, M. Piens, X. Wu, H. Pan, X. Hu, C. Gandin, R.H. Lipsky, J.C. Plumier, A.M. Marini, C. Heurteaux, Subchronic alpha-linolenic acid treatment enhances brain plasticity and exerts an antidepressant effect: a versatile potential therapy for stroke, Neuropsychopharmacology : official publication of the American College of Neuropsychopharmacology 34 (2009) 2548-2559.

[59] G. Pignataro, A. Scorziello, G. Di Renzo, L. Annunziato, Post-ischemic brain damage: effect of ischemic preconditioning and postconditioning and identification of potential candidates for stroke therapy, FEBS J 276 (2009) 46-57.

[60] A.M. Stowe, T. Altay, A.B. Freie, J.M. Gidday, Repetitive hypoxia extends endogenous neurovascular protection for stroke, Ann Neurol 69 975-985.

[61] N. Blondeau, R.H. Lipsky, M. Bourourou, M.W. Duncan, P.B. Gorelick, A.M. Marini, Alpha-Linolenic Acid: An Omega-3 Fatty Acid with Neuroprotective Properties: Ready for Use in the Stroke Clinic?, BioMed Research International (2014). 


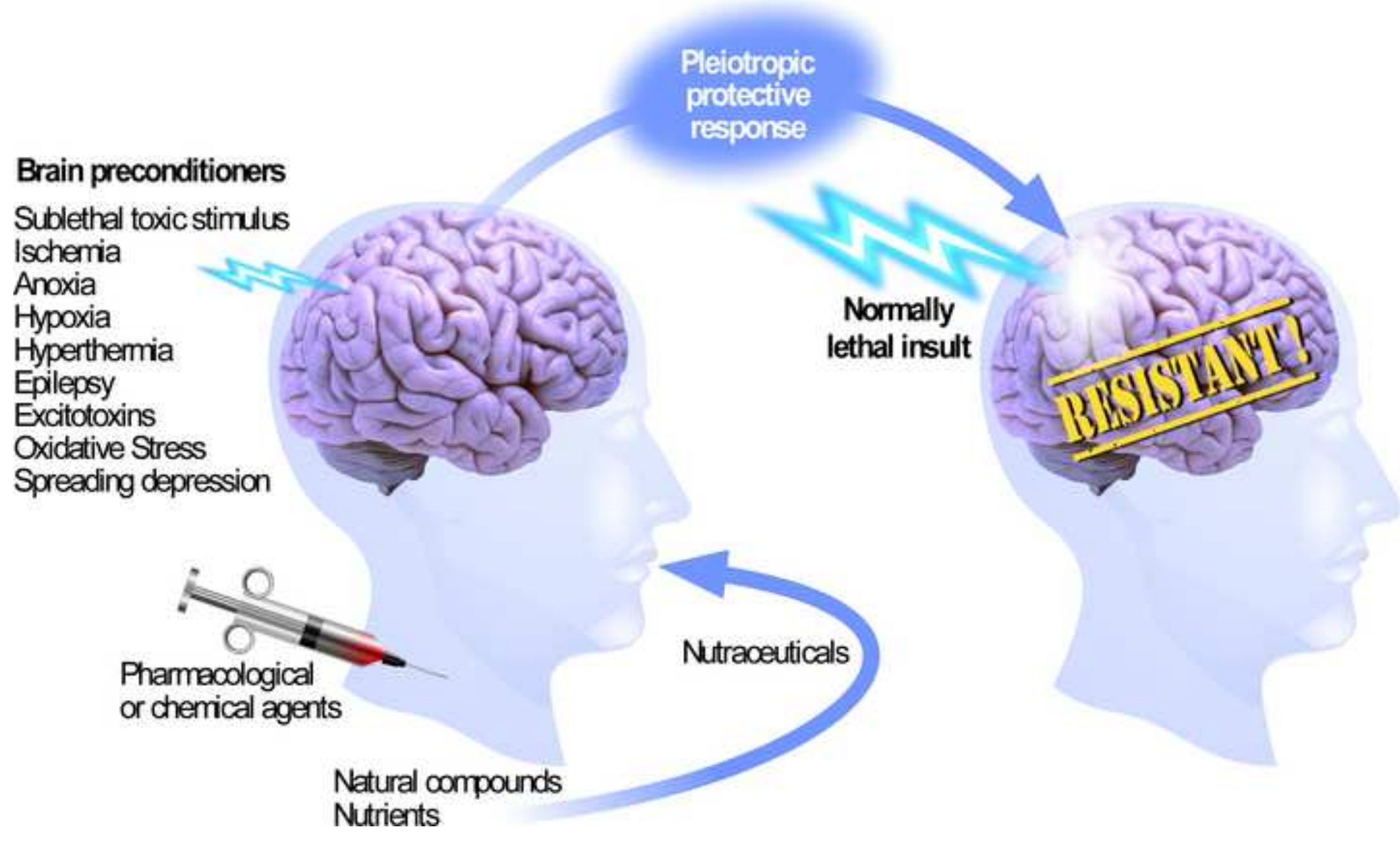

Nutrients

\section{Pieiotropic \\ protective \\ response}

Brain preconditioners

Ischerria

Anoxia

Hypoxia

Epilepsy

Excitotoxins

Oxidalive Stress

Spreading depression

ethal insult 
Nutritional approach

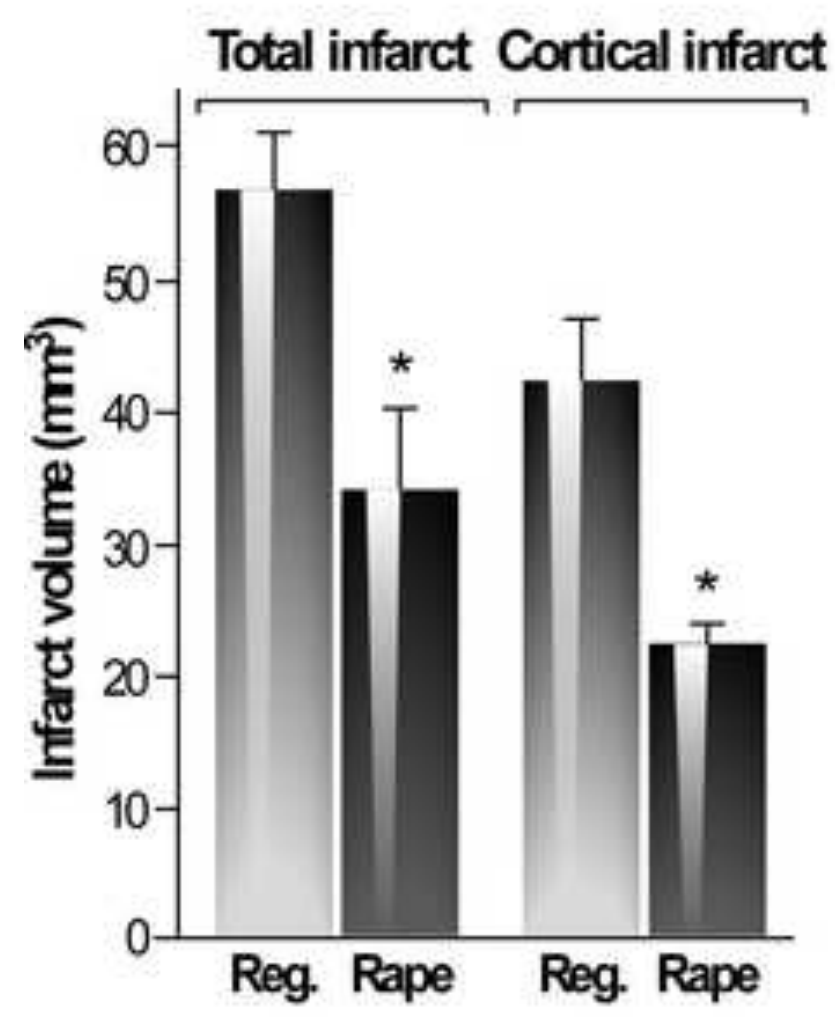

Medicinal approach

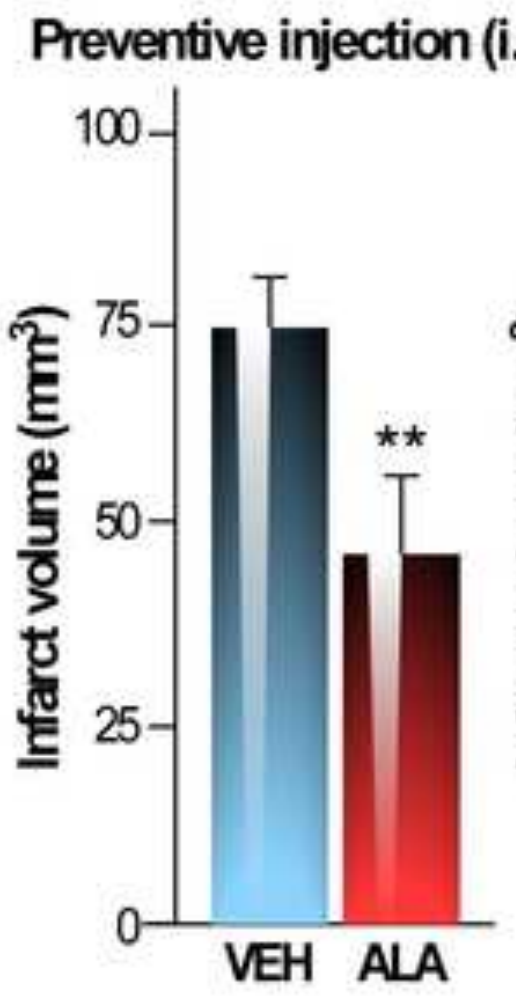

Post-treatment (i.v.)

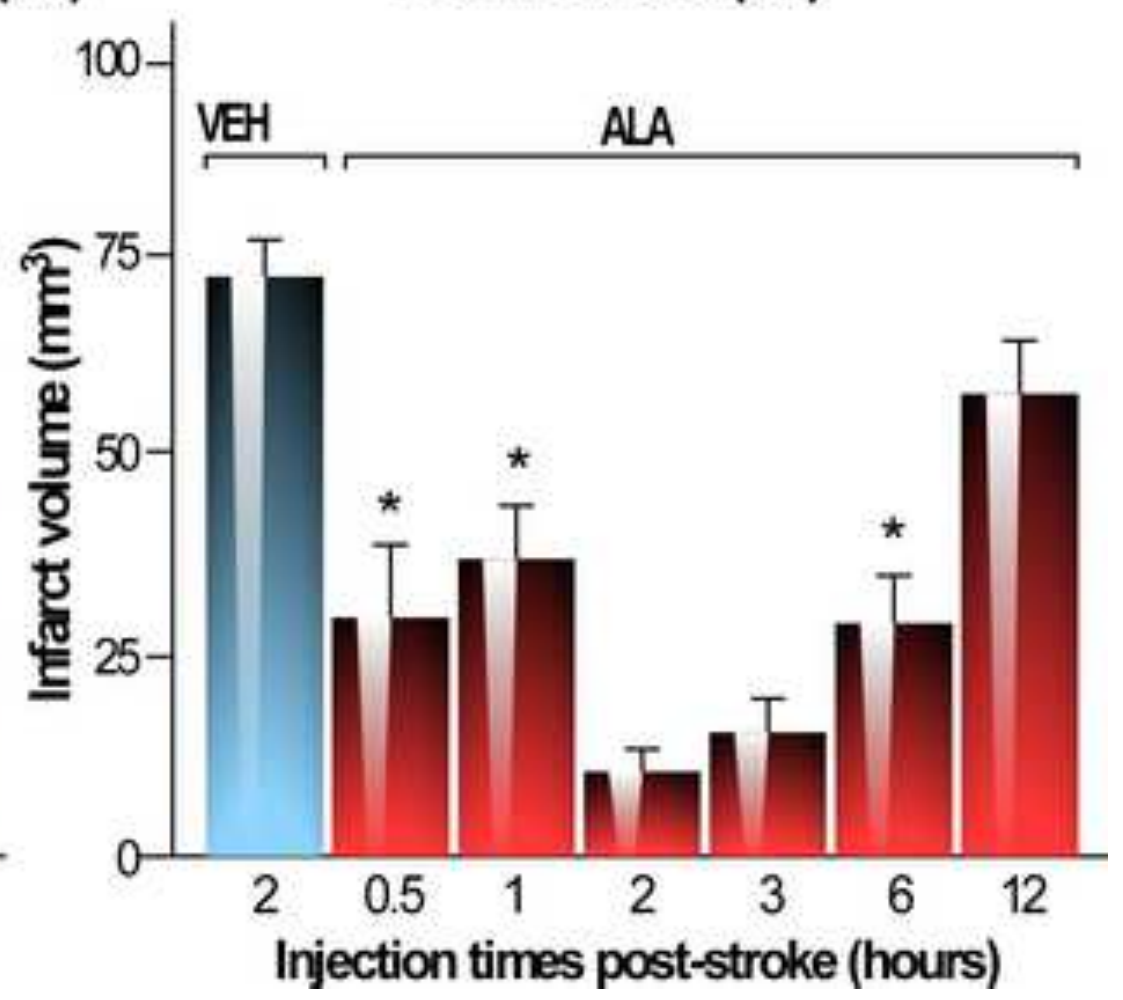

